\title{
Conformations of Polymers with Random Branches of Constant Number in Athermal Isolated Condition
}

\author{
Shujiro SHIGA ${ }^{\dagger}$ \\ Niihama National College of Technology, Niihama, Ehime 792, Japan
}

(Received December 4, 1996)

\begin{abstract}
Computer simulations were carried out for self-avoiding walk conformations of randomly branched polymers on a simple cubic lattice. The number of segments $(N)$ and branching points $(m)$ are set constant. The maximum $N$ and $m$ examined are 500 and 5, respectively. No attractive energy among the segments is considered. The standard success-walk number is 100000 for every condition. A critical exponent $(v)$ on $N$ for the root of the mean square radius of gyration $\left(\left\langle s^{2}\right\rangle^{1 / 2}\right)$ is found to be 0.598 . This $v$ is the same value as those of both linear and star-branched polymers reported in other papers. The $g$-values $\left(=\left\langle s^{2}\right\rangle_{\text {branch }} /\left\langle s^{2}\right\rangle_{\text {linear }}\right)$ obtained are nearly equal to those of the random walk in the $m$-range of this work. Generalization of the conclusion is speculated. $v$ of the case where $m$ distributes statistically is estimated to be 0.35 which is smaller by about 0.1 than that of other randomly-branched polymers. The difference, 0.1 , might be attributed to that of the distribution of branching points. The above description assumes the polymers long and flexible.
\end{abstract}

KEY WORDS Simulation / Excluded Volume Effect / Critical Exponent / $g$-Value / Distribution / Statistical / Animals / Trees /

Since 1995, the author and his coworkers have written papers on the conformation simulations of randomly branched polymers with excluded volume effect. The first in $1995^{1}$ reported on simplified simulation to reduce computing time. Verification of a simulation method of flexible randomly branched polymers ${ }^{2}$ was discussed in 1996. Rapid communication letters on simulation by this method for cases with and without interactions among segments ${ }^{3,4}$ will be published in the near future.

Zimm and Stockmayer ${ }^{5}$ studied random walk(RW) or the ideal conformations of polymers where there is no excluded volume effect. They concluded that the critical exponent $(v)$ is 0.5 , indifferent to the branching structure of the polymers, i.e., the same among linear, starshaped, comb-shaped and randomly branched polymer as long as the subchain number remains constant, while $v$ decreases to 0.25 if there exists not only the above variety of branching structure but also random distribution in the subchain number.

Some approaches have been used to solve conformations allowing the excluded volume effect, i.e., conformations with self-avoiding walk(SAW). LeGuillou and Zinn-Justin ${ }^{6}$ obtained 0.588 for $v$ according to their ferromagnetic body analogy. Miyake and Freed $^{7}$ succeeded in evaluating the excluded volume effect of linear and star-branched polymers and afterwards, Miyake ${ }^{8}$ evaluated the comb-branched polymers. The result was $v=0.5625$ for all. Last year, Cui and Chen ${ }^{9,10}$ solved the scaling rule of the polymers branched randomly on the structure and branching points. References 7-10 use the conformational space renormalization group theory. However, as they pointed out, higher order terms of $\varepsilon$-expansion should be estimated to get more precise results for the randomly branched polymers, even though very difficult and rather useless. Lubensky and Isaac$\operatorname{son}^{11,12}$ reported the results on the randomly branched polymers using ferromagnetic body analogy before Cui and Chen. The results of both groups are not very dif- ferent from each other. The conclusion $v=5 /[2(d+2)]$ was obtained by Lubensky and Isaacson ${ }^{13}$ using the Flory-type field theory and is believed more reliable than those of modern theories.

There are many papers on simulation. McCrackin et al. ${ }^{14}$ worked on linear polymers; Wittington et al. ${ }^{15}$ Kolinski and Sikorski, ${ }^{16}$ and Croxton ${ }^{17}$ on star-shaped polymers; Gallacher and Windwer ${ }^{18}$ worked on regular comb-shaped polymers. All $v$ obtained are nearly equal to 0.6 , irrespective of linear, star-shaped or comb-shaped polymers.

As far as the author is aware, there have been no published studies on the simulation of the randomly branched polymers with a constant number of branching points. However, there exist many works on polymers branched randomly both on structure and branching points. The following is summary for three dimensions.

$\operatorname{Redner}^{19}$ obtained $v=0.45$. Alexandrowicz ${ }^{20}$ published $v=0.42$ for Model S and 0.47 for Model T. Cui and $\mathrm{Chen}^{21}$ reported $v=0.49$ for the annealed branching structure and $v=0.45$ for the quenched branching structure by an off-lattice Monte Carlo method. For lattice trees, van Rensburg and Madras $^{22}$ obtained $v=0.496$; Seiz and Klein ${ }^{23} v=0.46$. It should be noted that the number of branches at branching points for the lattice trees are distributed in the range $3-6$.

The general relationship between $g$-factors and branching probability is not known by such simulations. Published $v$ incorporate not only the excluded volume effect but also branch distribution effect. The latter is caused by deviation from ideal statistical distribution of the branching points.

In this paper, long flexible polymers with fixed numbers of randomly branching points are studied instead of those of constant branching probability. This may allow us to separately estimate the previous two effects. 


\section{COMPUTATIONAL PROCEDURE}

\section{Computers, Programming Language, and Calculation}

The work stations, ews01 and alpha01, and the super computer, sx-3, of the Computation Center Osaka University were used for calculations. The programming language is $\mathrm{C}$. For each configution, 100000 conformations were calculated on a simple cubic lattice. Here, one configuration means one set of numbers of total segments and branching points of the polymer.

Until now, to carry out simulation, four procedures have been used: direct Monte Carlo (DMC) method, and the methods of Rosenbluth and Rosenbluth, ${ }^{24}$ Alexandrowicz, ${ }^{25}$ and Meirovitch. ${ }^{26}$ The author and his coworkers have used their Chain-Coupling method. ${ }^{2}$ The principle of the Alexandrowicz method was applied here not only to the elongation of the chain length but also to the chain-branching mechanism. This will be explained later in more detail in the chapter on algorithm.

\section{Algorithm for Conformation Simulations of Polymers}

Long Linear Polymers. Simulations were carried out for SAW on a simple cubic lattice, where it is prohibited to walk reversely and intersectingly on the lattice. Each walking direction is decided on by a random number. When a new walk violates the prohibition rule, the simulation is abandoned, and the next one starts from the origin. This is the DMC method.

The computing time would become intolerably long when using the DMC method if the chain sequence becomes very large. The time increases exponentially with increase in the walks. As a criterion, one hundred walks would be the limit of the DMC method. Alexandrowicz's method is adopted when this limit is exceeded. The procedure can be understood by the following example:

Let us realize linear 199 SAW's. First, the walks are divided into 100 and 99 walks. The two partial chains make their own conformations independently by the DMC method. The two conformations are connected to each other at the ends, and checked whether there are any commonly occupied lattice points.

If there are such common points, the connection is abandoned. In the case of a not linear but randomly branched polymer, the summed-up number of abandoned connections is necessary to obtain the conformationgenerating probability of each branching configuration which is the arrangement of the branching points of the polymer. Different configurations give the different conformation-generating probability.

Now let us return to the linear polymer. If the connection results in overlapping of the two next-toconnecting-end points of each partial chain, the abandonment number is not added one because of the prohibition rule on reverse growth of chain. To realize 299 walks, $100+100+99$ walks may be successively carried out.

The reason why the Alexandrowicz method gives a long chain with a rather small number of trials is attributed to the small looping chance of a long chain contrary to the large chance of a short chain as understood by the fractalic property of polymers.

Long Randomly-Branched Polymers. Procedure for obtaining long randomly-branched polymers is itemized as follows:

1. Let us consider a linear chain or polymer consisting of $N$ segments and cut it randomly into $2 m+1$ subchains. Here, $m$ corresponds to the number of branching points of a randomly branched polymer; the subchain to a sequence of the segments between the two neighboring branching points (an inner subchain) or between the free end and the neighboring branching point (an outer subchain).

A branching point has three subchains in this report; that is, functionality is three. This restriction is not essential and may not prevent generalization of this work.

The initial $m$-1 subchains become inner subchains, and the remaining the outer subchains. The bonding order is determined by the scission row of the linear chain. Because the scission is random, this bonding rule does not affect the randomness of the branched polymer.

2. Every subchain walks by SAW, the coordinates of which are memorized in a file. If the subchain is longer than 100 segments, the conformation will be made using the Alexandawicz method discribed in preceding section in Long Linear Polymers. Every subchain is connected to make the branched polymer one after another, according to the above-mentioned bonding order.

Looping or intersecting, which is against SAW, is checked for all lattice points. If the rule is infringed on, the conformation is abandoned and one is added to the trial number, except when the two next-to-connecting-point segments occupy the same lattice point as stated in the preceding section. The successfully bonded subchain is memorized via the coordinates of the constituting segments.

3. The success probability of preparing a randomly branched polymer is calculated as follows:

First, success probability for every subchain is obtained from the trial number. Second, the success probability for every bonding is similarly obtained. Third, these partial probabilities are multiplied by one another to obtain the necessary probability. The partial probabilities are mutually independent.

The above is an expanded Alexandrowicz method and was named the Chain-Coupling method in our previous paper. ${ }^{2}$ This method requires weighting of the ease of preparing conformations different from each other by configuration. The need for this weighting is caused by different subchain-lengths and different branching point distributions, both of which result in different preparation probability for each configuration, even if $N$ and $m$ are the same.

300000 conformations of subchains per a combination of $N$ and $m$ are the standard.

\section{RESULTS AND DISCUSSION}

\section{Critical Exponent of Constant Branching Point Polymers}

Table I shows RW and SAW results of polymers with various total chain-lengths and various numbers of branching points.

The 5-choice type RW of a linear chain theoretically gives

$$
\left\langle s^{2}\right\rangle_{1}=0.25 N
$$


and the experimental values coincide almost completely with eq 1 . The $\left\langle s^{2}\right\rangle$ of $\mathrm{RW}$ of a randomly branched chain can be expressed as

$$
\left\langle s^{2}\right\rangle_{\mathrm{br}}=A(m) N
$$

where infinity is assumed for $N$. Kurata and Fukatsu ${ }^{27}$ derived $g$-values for the branched polymers. Here, $g$ indicates the ratio of the mean square radius of gyration of a branched polymer $\left\langle s^{2}\right\rangle_{\mathrm{br}}$ to that of a linear polymer with the same molecular weight $\left\langle s^{2}\right\rangle_{1}$. They give $A(m)$ 's of $m=1,2,3$, and 5 to be $0.225,0.207,0.194$, and 0.173 , respectively. Table I shows that the simulation values almost coincide with the theoretical ones. The longer the polymer chain, the better the coincidence.

McCrackin et al. concluded the following equation from their SAW simulations of linear polymers in a simple lattice:

$$
\left\langle s^{2}\right\rangle_{1}=0.170 N^{2 \times 0.598}
$$

The range of $N$ is $15-150$. The coincidence between eq 3 and the SAW data in Table I is excellent.

The least square fitting of the data for the branched polymers gives 0.5964 as the average of the critical exponent, which is practically equal to the above 0.598 . Therefore, again, the relationship of branched polymers can be expressed by the following:

$$
\left\langle s^{2}\right\rangle_{\mathrm{br}}=B(m) N^{2 \times 0.598}
$$

$B(m)$ 's for $m=1,2,3$, and 5 are $0.154,0.143,0.134$, and 0.121 , respectively.

\begin{tabular}{|c|c|c|c|}
\hline$N$ & $m$ & $\left\langle s^{2}\right\rangle^{\mathrm{a}}$ & $\left\langle s^{2}\right\rangle^{\mathrm{a}}$ \\
\hline $\begin{array}{l}\text { Total chain } \\
\text { length }\end{array}$ & $\begin{array}{l}\text { No. of branching } \\
\text { points }\end{array}$ & SAW & RW \\
\hline 50 & 0 & $1.83 \mathrm{e}+01$ & $1.25 \mathrm{e}+01$ \\
\hline 50 & 1 & 1.66 & 1.11 \\
\hline 50 & 2 & 1.53 & 1.01 \\
\hline 50 & 3 & 1.43 & 0.930 \\
\hline 100 & 0 & 4.19 & 2.49 \\
\hline 100 & 1 & 3.81 & 2.24 \\
\hline 100 & 2 & 3.58 & 2.01 \\
\hline 100 & 3 & 3.29 & 1.89 \\
\hline 100 & 5 & 2.99 & 1.66 \\
\hline 200 & 0 & 9.61 & 4.96 \\
\hline 200 & 1 & 8.61 & \\
\hline 200 & 2 & 8.01 & 4.11 \\
\hline 200 & 3 & 7.56 & 3.82 \\
\hline 200 & 5 & 6.86 & 3.38 \\
\hline 500 & 0 & $2.83 e+02$ & $1.24 \mathrm{e}+02$ \\
\hline 500 & 1 & 2.60 & 1.12 \\
\hline 500 & 2 & 2.40 & 1.03 \\
\hline 500 & 3 & 2.26 & 0.961 \\
\hline 500 & 5 & 2.024 & 0.851 \\
\hline
\end{tabular}

Table I. Conformations of randomly branched polymers in a simple cubic lattice

${ }^{\mathrm{a}}$ Mean square radius of gyration.

Table II. Comparison of $g$-factors of RW with those of SAW

\begin{tabular}{lccccc}
\hline $\begin{array}{c}m \\
\text { No. of branching points }\end{array}$ & 0 & 1 & 2 & 3 & 5 \\
\hline$g$ of RW & 1.000 & 0.900 & 0.828 & 0.776 & 0.692 \\
$g$ of SAW & 1.000 & 0.907 & 0.843 & 0.788 & 0.714 \\
$(g$ of RW $) /(g$ of SAW $)$ & 1.000 & 0.992 & 0.982 & 0.985 & 0.969 \\
\hline
\end{tabular}

In Table II, $g$ of SAW calculated using Table I are compared with those of RW. The coincidence between the two groups is delicate. The formers might become gradually larger than the latters with increase of $m$. Simulations with larger $m$ would be looked for the strict conclusion.

Large $m$ and $N$, especially $m$, makes the computation very difficult because of long cpu time and large memory required. The last calculation of the scheme, $m=5$ and $N=500$, consumed actually about 2.5 months, nearly the same as for the sum for the others, by the timesharing computer alpha01 of multi-client service. Another algorithm suitable to larger $m$ should be developed.

In the succeeding section, the author likes to proceed with the discussion under the probable speculation that the two $g$-values coincide with each other for the same $m$. Mazur and McCrackin ${ }^{28}$ arrived at a similar conclusion for the star-shaped polymers. The equality of the two $g$ s will then be common for all branched polymers regardless of the types of branching if the polymers are long and flexible enough to avoid becoming rigid and/or wormlike. Extremely high density of branching points will result in such polymers, which is beyond the scope of this study.

There is a not negligible discrepancy between simulated values of the critical exponent by Mazur et al. and theoretical values by Miyake et al., who derived their formula by the first-term approximation of the $\varepsilon$-expansion just as Cui et al. did. Therefore, the simulated values may be more important for the purpose of this study.

\section{Critical Exponent for Polymers with Branching Points of Statistical Distribution}

All simulations reported for randomly branched polymers are carried out under the condition of fixed segment numbers and fixed branching probabilities. These would be closely related with the above results which come out of the condition of fixed numbers of branching points instead of fixed branching probabilities. Let us consider the case where branching occurs ideally, that is, the polymer obeys RW.

Shultz $^{29}$ investigated the case and formulated the following relationship:

$$
p(m \mid Z)=\left(\gamma^{1 / 2} Z\right)^{2 m+1} / m ! /(m+1) ! / I_{2}\left(2 \gamma^{1 / 2} Z\right)
$$

Here, $Z, \gamma$, and $I_{2}$ indicate reduced molecular weight, branching index and the modified Bessel function of the second order, respectively. The mean structural properties of the polymer of the previous distribution may be obtained by weighting $p(m \mid Z)$ individually on the constituents of the polymer and summing them up for $m=0-\infty$. Hence, the average number of the branching points $\langle m\rangle$ is given by

$$
\langle m\rangle=\sum m p(m \mid Z)
$$

and average of $\left\langle s^{2}\right\rangle_{\mathrm{br}}$ for the distribution by

$$
\left\langle\left\langle s^{2}\right\rangle_{\mathrm{br}}\right\rangle=\sum\left\langle s^{2}\right\rangle_{\mathrm{br}} p(m \mid Z)
$$

Equation 7 was approximately solved by Zimm and Stockmayer as 
$\left\langle\left\langle s^{2}\right\rangle_{\mathrm{br}}\right\rangle=\left\langle s^{2}\right\rangle_{1}\left\{[1+(\langle m\rangle / 7)]^{1 / 2}+(4\langle m\rangle / 9 \pi)\right\}^{-1 / 2}$

where $\left\langle s^{2}\right\rangle_{1}$ is given by eq 1 . Because the branching index $\gamma$ is a constant, the value inside the parentheses \{\} depends on the second term for large $Z$ or $N$. Therefore, $\left\langle\left\langle s^{2}\right\rangle_{\text {br }}\right\rangle$ is proportional to $N^{1 / 2}$, and $\langle A(m)\rangle$ inversely proportional to $N^{1 / 2}$. Here, $\langle A(m)\rangle$ is the average coefficient $A(m)$ of eq 2 as follows:

$$
\langle A(m)\rangle=\sum A(m) p(m \mid Z)
$$

As suggested in the preceding section, $B(m)$ for a SAW chain might be speculated as almost proportional to the $A(m)$ for the corresponding RW chain, so that $\left\langle\left\langle s^{2}\right\rangle_{\text {br }}\right\rangle$ would be also proportional to $N^{1.196-1 / 2}=$ $N^{0.7}$. That is, $v$ would be equal 0.35 .

As written in the introduction, $v \sim 0.45$ has been obtained by several investigators who carried out simulations on the randomly branched polymers having distribution in number of branching points. The difference $0.45-0.35=0.1$ arises from the discrepancy of the branch distribution between RW and SAW by the excluded volume effect.

The branched chains in the references slightly differ from one another in randomness. In Alexandrowicz's model S, a growing tip of a branched polymer is randomly chosen and a bifunctional or polyfunctional monomer becomes irreversibly attached to it. In model $\mathrm{T}$, termination of growth in the former model is assumed to happen with some probability. These models were proposed as the criticism for the construction of an ensemble of animals to be at equilibrium with respect to the bonds' rearrangement. Redner achieved his work on the model S. Cui et al.'s annealed branched structures are generated with constant branching activities and belong to the systems where rearrangement of branching points may take place. In their quenched branched structures, the introduction of monomer-monomer interaction leads to stretching of the polymer, but does not affect the structures themselves.

It is of interest to know how the distribution of branching points appears and influences the $v$ as a result of the excluded volume effect, although more detailed discussion than the above might be difficult because, until now, such investigations have been seldom published.
Acknowledgment. The author expresses his appreciation to the members of the Computation Center Osaka University for their helpful suggestions on the computation.

\section{REFERENCES}

1. S. Shiga, N. Oka, T. Shirasaya, and T. Hongu, Niihama Kogyo Koto Senmongakko Kiyo (Rikogaku Hen), 31, 76 (1995).

2. S. Shiga, N. Oka, K. Takahashi, and T. Fujii, Niihama Kogyo Koto Senmongakko Kiyo (Rikogaku Hen), 32, 102 (1996).

3. S. Shiga, N. Oka, and K. Shiomi, Niihama Kogyo Koto Senmongakko Kiyo, 33, 93 (1997).

4. S. Shiga, M. Kogami, M. Tanaka, and K. Yano, Niihama Kogyo Koto Senmongakko Kiyo, 33, 88 (1997).

5. B. H. Zimm and W. H. Stockmayer, J. Chem. Phys., 17, 1301 (1949).

6. J.C. LeGuillou and J. Zinn-Justin, J. Phys. Rev. B, 21, 3976 (1980).

7. A. Miyake and K. F. Freed, Macromolecules, 16, 1228 (1983).

8. A. Miyake, Preprints, Japan-US Polymer Symposium, Kyoto, Japan, organized by the Society of Polymer Science, Japan, Tokyo, 1985, p 273.

9. S.-M. Cui and Z. Y. Chen, Phys. Rev. E, 52, 3943 (1995).

10. S.-M. Cui and Z. Y. Chen, Phys. Rev. E, 52, 5084 (1995).

11. T. C. Lubensky and J. Isaacson, Phys. Rev. A, 20, 2130 (1979).

12. T. C. Lubensky and J. Isaacson, Phys. Rev. Lett., 41, 829 (1978).

13. J. Isaacson and T. C. Lubensky, J. Phys. Paris, Lett., 41, L469 (1980).

14. F. L. McCrackin, J. Mazur, and C. M. Guttman, Macromolecules, 6, 859 (1973).

15. S. G. Wittington, J. E. G. Lipson, M. K. Wilkinson, and D. S. Gaunt, Macromolecules, 19, 1241 (1986).

16. A. Kolinski and A. Sikorski, J. Polym. Sci., Polym. Chem. Ed., 20, 3147 (1982).

17. C. A. Croxton, Macromolecules, 21, 2269 (1988).

18. L. V. Gallacher and S. Windwer, J. Chem. Phys., 44, 1139 (1966).

19. S. Redner, J. Phys. A, Math. Gen., 12, L239 (1979)

20. Z. Alexandrowicz, Phys. Rev. Lett., 54, 1420 (1985)

21. S.-M. Cui and Z. Y. Chen, Phys. Rev. E, 53, 6238 (1996).

22. E. J. Jannse van Rensburg and N. Madras, J. Phys. A: Math. Gen., 25, 303 (1992).

23. W. A. Seitz and D. J. Klein, J. Chem. Phys., 75, 15 (1981).

24. A. W. Rosenbluth and M. N. Rosenbluth, J. Chem. Phys., 23, 356 (1955).

25. Z. Alexandrowicz, J. Chem. Phys., 51, 561 (1969).

26. H. Meirovitch, J. Phys. A, Math. Gen., 15, L735 (1982).

27. M. Kurata and M. Fukatsu, J. Chem. Phys., 41, 2934 (1964).

28. J. Mazur and F. McCrackin, Macromolecules, 10, 326 (1977).

29. A. R. Shultz, J. Polym. Sci., A, 3, 4211 (1965). 